\title{
Article
}

\section{Validation of the Eating Disorder Examination Questionnaire in Danish Eating Disorder Patients and Athletes}

\author{
Mia Beck Lichtenstein 1,2,*, Lauge Haastrup ${ }^{2}$, Karen Krogh Johansen ${ }^{2}$, Jacob B. Bindzus ${ }^{2}$, Pia Veldt Larsen ${ }^{3}$, \\ René Klinkby Støving ${ }^{4}$, Loa Clausen ${ }^{5,6}{ }^{\infty}$ and Jakob Linnet ${ }^{2}$ \\ 1 Department of Clinical Research, University of Southern Denmark, 5000 Odense C, Denmark \\ 2 Centre for Telepsychiatry, Mental Health Services in the Region of Southern Denmark, \\ 5000 Odense C, Denmark; laugehaastrup@gmail.com (L.H.); Karen.krogh.johansen@rsyd.dk (K.K.J.); \\ jacob.bindzus@outlook.dk (J.B.B.); Jakob.Linnet@rsyd.dk (J.L.) \\ 3 Mental Health Services in the Region of Southern Denmark, 5000 Odense C, Denmark; \\ pia.veldt.larsen@rsyd.dk \\ 4 Center for Eating Disorders, Odense University Hospital, 5000 Odense C, Denmark; rene.stoeving@rsyd.dk \\ 5 Department of Child and Adolescent Psychiatry, Aarhus University Hospital, 8200 Aarhus N, Denmark; \\ loaclaus@rm.dk \\ 6 Department of Clinical Medicine, Aarhus University, 8200 Aarhus N, Denmark \\ * Correspondence: m.lichtenstein@health.sdu.dk; Tel.: +45-2621-8846
}

Citation: Lichtenstein, M.B.; Haastrup, L.; Johansen, K.K.; Bindzus, J.B.; Larsen, P.V.; Støving, R.K.; Clausen, L.; Linnet, J. Validation of the Eating Disorder Examination Questionnaire in Danish Eating Disorder Patients and Athletes J. Clin. Med. 2021, 10, 3976. https:// doi.org/10.3390/jcm10173976

Academic Editors: Elena Tomba, Yael Latzer, Daniel Stein and Itay Tokatly Latzer

Received: 7 July 2021

Accepted: 10 August 2021

Published: 2 September 2021

Publisher's Note: MDPI stays neutral with regard to jurisdictional claims in published maps and institutional affiliations.

Copyright: (c) 2021 by the authors. Licensee MDPI, Basel, Switzerland. This article is an open access article distributed under the terms and conditions of the Creative Commons Attribution (CC BY) license (https:// creativecommons.org/licenses/by/ $4.0 /)$.

\begin{abstract}
The Eating Disorder Examination Questionnaire (EDE-Q) is a gold standard questionnaire to identify eating disorder symptoms but has not yet been validated in Danish. The scale consists of four theoretical constructs of disordered eating: Restraint eating, Eating concerns, Shape concerns and Weight concerns. However, the four-factor structure has been difficult to replicate across cultures. This study aimed to examine the factor structure and psychometric properties of the EDE-Q in Danish. The study consisted of four samples (aged 15-70): Patients with anorexia, bulimia and unspecified eating disorders $(n=101)$, patients with symptoms of binge-eating disorder $(n=300)$, recreational athletes $(n=404)$, and elite athletes $(n=526)$. Depending on the analysis performed, participants had to complete the EDE-Q, the SCOFF questionnaire for eating disorders or the Binge Eating Disorders Questionnaire. In accordance with international research, we found no evidence for a four-factor structure in the EDE-Q among patients or among athletes. But our results showed significant, positive associations between EDE-Q and SCOFF, BED-Q and MDI in all samples. We conclude that the internal structure of EDE-Q is low, while construct validity is high, making EDE-Q useful as an instrument to identify individuals with eating disorder symptoms, including recreational, and elite athletes.
\end{abstract}

Keywords: Eating Disorder Examination Questionnaire; eating disorders; validation; factor structure; exercise; sport

\section{Introduction}

The Eating Disorder Examination Questionnaire (EDE-Q), originally developed by Fairburn and Beglin [1], measures behavioral and cognitive symptoms of eating disorders, including binge eating, self-induced vomiting, excessive exercise and negative body image [2].

The questionnaire is based on the Eating Disorder Examination Interview (EDE-I), which is the gold standard in eating-disorder assessment. The EDE questionnaire (EDE-Q) was developed to provide a straightforward and quick assessment tool and is one of the most used instruments to screen for symptoms of eating disorders [3]. It consists of four subscales measuring 1. Restraint, 2. Eating concern, 3. Shape concern, and 4. Weight concern. The EDE-Q has a global score that is an average of the four subscales and is used as a cut-off point for eating-disorder pathology. 
The scale has been validated across several different languages. Berg et al. found that the EDE-Q had acceptable psychometric properties in a literature review of Englishspeaking adult samples from different countries [2]. Other studies have examined the reliability or validity of the EDE-Q in Spanish [4-6], Japanese [7], Turkish [8], Persian [9], Finnish [10], Hebrew [11], Greek [12], Norwegian [13,14], Swedish [15], French [16] and German [17]. However, the EDE-Q has never been validated in Danish. A Danish validation of the EDE-I was conducted by Kjeldberg et al. [18] based on 1586 participants from a clinical eating-disorder sample (all female). An exploratory factor analysis was conducted and suggested both one-, two- and three-factor models, depending on the sub-sample. The factor of Food Restraint seemed consistent among the groups.

The original theoretical four-factor structure of EDE-Q has been questioned in several empirical studies. A review by Berg et al. found three studies evaluating the factor structure of the EDE-Q, none supporting the original four-factor model [2]. One of the studies compared the original four-factor model to a three-, two- and one-factor model and found the one-factor solution to be the best fit. Another study found a three-factor solution consisting of 1 . Dietary Restraint, 2. Shape/Weight Over-evaluation and 3. Body Dissatisfaction. The third study found support for a two-factor model, consisting of a Restraint factor and a combined Eating-Shape-Weight concern factor.

The EDE-Q factor structure has been examined empirically since the review by Berg et al., but the studies have not been reviewed and synthesized [2]. Furthermore, the factor structure of EDE-Q has not been evaluated in Danish yet.

Most studies report a higher prevalence proportion of eating disorder symptoms among athletes compared to the general population $[19,20]$. While several studies have used the EDE-Q to assess the frequency of eating disorders in athletes, only one study has validated the EDE-Q in sport [21]. The authors concluded that a three-factor solution provided the best fit for all groups, except for the sedentary males. They also emphasized that athletes may display the same behaviors towards weight and shape as sedentary people but differ according to diet and eating concerns. More research investigating the validity of the EDE-Q in athletic populations is requested.

The main aim of this study was to conduct a confirmatory factor analysis of the EDE-Q in a Danish sample of patients with eating disorders and a sample of recreational and elite athletes. Secondly, we aimed to assess the construct validity of EDE-Q by comparing the EDE-Q global score with other measures of eating pathology and depression. Based on existing research we expected the factor structure of EDE-Q to be inconsistent, but we assumed the instrument had a modest-to-high positive correlation with other measures of eating disorders and depression.

Before conducting the psychometric analyses we reviewed the literature testing factor models of the Eating Disorder Examination Questionnaire (EDE-Q).

\section{Materials and Methods}

The review study was conducted as a literature review (or rapid review) using the following search to create a brief overview of the findings of factor models: EDE-Q AND Athlete*s OR sport athlete*s AND eating disorder* OR anorexia nervosa OR bulimia nervosa OR binge eating disorder OR unspecified eating disorder AND factor analysis OR factor exploration. We only included English language publications in the period 2012-2021.

The empirical research design was a questionnaire-based cross-sectional data-collection consisting of four sub-samples:

1. Individuals with Anorexia Nervosa (AN), Bulimia Nervosa (BN), and unspecified eating disorders;

2. Individuals seeking treatment for mild-to-moderate Binge Eating Disorder (BED) in a Mental Health Department;

3. Elite athletes competing at the highest national and international level;

4. Recreational athletes practicing physical activity on a weekly basis. 


\subsection{Participants}

The first subsample consisted of 101 individuals with self-reported AN $(n=55)$, BN $(n=10)$, and unspecified eating disorders $(n=36)$ recruited from national patient organizations, communal health services or patients admitted to psychiatric wards in Denmark. Recruitment occurred during April 2020 and November 2020.

The second subsample included 300 participants seeking a public internet-based treatment program for mild to moderate BED. Participants were recruited between May 2019 and November 2020.

The third subsample consisted of 404 Danish elite athletes across 15 different sports; the most common were cycling, track and field, handball, football, karate, swimming, triathlon and gymnastics. The participants completed the questionnaire between December 2019 and March 2020 and were part of a large cross-sectional study on mental health in Danish elite athletes [22].

The fourth subsample consisted of 526 Danish recreational athletes recruited through social media channels between March and May 2020. A description of the study including a link to the survey was posted on various exercise groups on Facebook. The most common sports were: running (recreational), fitness (power and endurance), track and field, handball, triathlon, cycling, gymnastics and boxing.

\subsection{Measures}

All participants completed the EDE-Q and the Major Depressive Inventory (MDI) in Danish. The sample of eating-disorder patients, recreational athletes, and elite athletes also completed the eating-disorder screening instrument, SCOFF. The BED sample completed the Binge Eating Disorder Questionnaire (BED-Q) developed in-house to assess BED symptoms. See Appendix A.

We used a Danish version of the EDE-Q, translated by Elsass et al. [23] with back translation approved by Christopher Fairburn. The EDE-Q has 28 items and four subscales measuring 1. Restraint, 2. Eating concern, 3. Shape concern and 4. Weight concern. A total score called "Global Score" is an average of the subscales.

SCOFF is a five-item screening tool designed to assess basic symptoms of BN and AN [24]. The measure is scored on a dichotomous "yes" or "no" scale and two or more positive answers indicate risk of an eating disorder. A meta-analysis of the diagnostic accuracy of the SCOFF scale showed excellent performance [25]. The SCOFF has been translated and validated in Danish showing reasonable sensibility and sensitivity [26].

The Binge Eating Disorder Questionnaire (BED-Q) was developed in-house to assess symptoms of BED. It is a 9-item scale based on the diagnostic criteria for BED in the Diagnostic and Statistical Manual of Mental Disorders (DSM-5) [27]. The BED-Q is attached as an appendix.

The MDI contains 10 items that cover symptoms of depression defined by the International Statistical Classification of Diseases and Related Health Problems 10th edition (ICD-10) [28]. Answers are given on a 6-point Likert scale, and the total score ranges from 0 to 50. Recommended cut-off points are 21 for mild depression, 26 for moderate depression, and 31 for severe depression [29]. The scale has been validated and shows good psychometric properties in several studies including Danish $[29,30]$.

\subsection{Ethics}

By completing the questionnaire, participants (or parents for participants aged 15-17 years) gave their informed consent for scientific use. No personal data, such as names, birthdays, or e-mail addresses were mandatory to complete the questionnaire. Data collection based on anonymous questionnaires does not need ethical approval, as confirmed by The Regional Committees on Health Research Ethics for Southern Denmark (approval number 20212000-57 and 20192000-145). 


\subsection{Statistical Analyses}

Analyses were performed in SPSS version 26 and STATA 16.1 (Statacorp, College Station, TX, USA). Descriptive statistics on participant characteristics were computed. Chi square analyses were used for dichotomous variables and $t$-tests were used for comparing mean scores.

The factor model comprising the four EDE-Q factors (Restraint (EDE-Q Items 1-5), Eating concern (Items 7, 9, 19, 20, 21), Shape concern (Items 6, 10, 11, 23, 26, 27, 28, Note: not including Item 8) and Weight concern (Items 8, 12, 22, 24, 25)) was analyzed in two combined samples: one combining the eating disorder and BED samples, and one combining the elite and recreational samples. Structural equation models (SEM) were used to conduct confirmatory factor analyses (CFA) with standard errors estimated using non-parametric bootstrap sampling with 1000 replications, to account for non-normality of the latent factors. Sensitivity analyses were conducted of the CFAs with Item 8 attributed to the factor on Shape concern rather than Weight concern.

The goodness of fit of the factor models was assessed by means of comparative fit index (CFI), Tucker-Lewis index (TLI), root-mean-square error of approximation (RMSEA), and standardized root-mean-square residual (SRMR). Further, Cronbach's $\alpha$ was used to assess the internal consistency of each of the four factors.

Construct validity was assessed by using Pearson and Spearman correlations between EDE-Q and other measures of psychopathology SCOFF/BED-Q and MDI.

\section{Results}

\subsection{Literature Review}

Our literature review resulted in 19 publications from 2012 to 2021 conducting a factor analysis of the EDE-Q. The studies are presented in Table 1 and show remarkable inconsistency across samples. There is no evident factor structure, and the studies display both one-, two- and three-factor models.

Table 1. Factor structure of the Eating Disorder Examination Questionnaire in cross-cultural samples in the period 2012-2021.

\begin{tabular}{|c|c|c|c|c|}
\hline Authors & Year & Country & Sample & Factor Solution and Item Number \\
\hline Barnes et al. [31] & 2012 & UK & $\begin{array}{c}569 \text { adult participants including } \\
403 \text { university students ( } 91.8 \% \\
\text { female) and } 166 \text { eating disorder } \\
\text { patients } \\
(95.8 \% \text { female). } \\
\text { Age is not mentioned. }\end{array}$ & $\begin{array}{l}\text { A three-factor model suggested by } \\
\text { Peterson et al. } 2007 \text { found best fit for } \\
\text { both groups: (1) Weight and shape } \\
\text { concern, (2) Eating concern and } \\
\text { (3) Restraint. }\end{array}$ \\
\hline Penelo et al. [32] & 2013 & Mexico & $\begin{array}{l}2928 \text { schoolchildren (1544 females, } \\
1384 \text { males), mean age } 15.2 \\
\text { (SD = 1.79) and } 15(\mathrm{SD}=1.78) \text { years } \\
\text { for females and males, respectively. }\end{array}$ & $\begin{array}{l}\text { A two-factor model was suggested } \\
\text { as the best fit: (1) A combination of } \\
\text { eating weight shape concerns and } \\
\text { (2) Restraint. }\end{array}$ \\
\hline Giovazolias et al. [12] & 2013 & Greece & $\begin{array}{c}664 \text { participants including } \\
500 \text { university students (all female), } \\
\text { mean age } 20.55(\mathrm{SD}=3.27) \text { years, } \\
\text { and } 164 \text { psychology students (all } \\
\text { female), mean age } \\
20.90(\mathrm{SD}=3.29) \text { years. }\end{array}$ & $\begin{array}{l}\text { Three-factor models were found to } \\
\text { have the best fit: (1) Combined } \\
\text { Shape- and Weight Concern factor, } \\
\text { (2) Restraint factor, and (3) Eating } \\
\text { Concern factor. }\end{array}$ \\
\hline Darcy et al. [21] & 2013 & USA & $\begin{array}{l}1637 \text { university students separated } \\
\text { into two groups: } 976 \text { competitive } \\
\text { athletes ( } 544 \text { female, } 432 \text { male) and } \\
858 \text { non-athletes ( } 429 \text { female, } \\
229 \text { male). Mean age } 20.87 \\
\text { (SD }=1.66 \text { ) years. }\end{array}$ & $\begin{array}{l}\text { A three-factor model provided the } \\
\text { best fit, except for non-athlete male } \\
\text { participants where the best fit was a } \\
\text { two-factor model. }\end{array}$ \\
\hline
\end{tabular}


Table 1. Cont.

\begin{tabular}{|c|c|c|c|c|}
\hline Authors & Year & Country & Sample & Factor Solution and Item Number \\
\hline Grilo et al. [33] & 2014 & USA & $\begin{array}{c}801 \text { University students ( } 573 \text { female, } \\
228 \text { male), mean age } \\
20(\mathrm{SD}=2.5) \text { years. }\end{array}$ & $\begin{array}{l}\text { Evidens for a seven-item, } \\
\text { three-factor model: (1) Dietary } \\
\text { restraint, (2) Shape/weight } \\
\text { overvaluation and (3) Body } \\
\text { dissatisfaction. }\end{array}$ \\
\hline White et al. [34] & 2014 & UK & $\begin{array}{c}\text { Two adolescent samples: }(1) \\
458 \text { participants }(257 \text { female, } \\
201 \text { male), mean age } 15.3(\mathrm{SD}=1.18) \\
\text { years, and (2) } 259 \text { participants } \\
\text { (265 female, } 194 \text { male), mean age } \\
15.2(\mathrm{SD}=1.18) \text { years. }\end{array}$ & $\begin{array}{l}\text { A three factor, 22-item model was } \\
\text { found as best fit: (1) Shape and } \\
\text { weight concerns, (2) Restriction and } \\
\text { (3) Preoccupation and eating } \\
\text { concern. }\end{array}$ \\
\hline Carrard et al. [16] & 2015 & France & $\begin{array}{c}116 \text { patients with BED (all female), } \\
\text { mean age } 38.5(\mathrm{SD}=11.4) \text {, and } \\
161 \text { participants without a eating } \\
\text { disorder (all female), mean age } \\
28.1(\mathrm{SD}=8.1 \text { ) years. }\end{array}$ & $\begin{array}{l}\text { A seven-item, three-factor model } \\
\text { suggested by Grilo et al. } 2013 \text { [33] } \\
\text { revealed an adequate fit. }\end{array}$ \\
\hline Kliem et al. [17] & 2016 & Germany & $\begin{array}{l}2520 \text { participants from } 2009 \text { study } \\
(1334 \text { female, } 1174 \text { male), mean age } \\
49.67(\mathrm{SD}=18.30) \text { and } 2508 \\
\text { participants from } 2013 \text { ( } 1354 \text { female, } \\
1166 \text { male), mean age } 49.90-51.02 \\
(\mathrm{SD}=18.20-18.66) \text { from the general } \\
\text { population. }\end{array}$ & $\begin{array}{l}\text { An eight-item with second-order } \\
\text { corrected factors found the best fit. }\end{array}$ \\
\hline $\begin{array}{l}\text { Forsén Mantilla et al. } \\
\qquad[15]\end{array}$ & 2017 & Sweden & $\begin{array}{l}487 \text { school children ( } 239 \text { female, } \\
248 \text { male), mean age } \approx 13.50 \\
\text { (SD }=0.50) \text { years, and } 570 \text { patients } \\
\text { with eating disorders (all female), } \\
\text { mean age } 13.46(\mathrm{SD}=0.69 \text { ) years. }\end{array}$ & $\begin{array}{l}\text { One underlying factor was found for } \\
\text { adolescent girls: Dissatisfaction with } \\
\text { shape and weight. For boys three } \\
\text { factors were found: } \\
\text { (1) Weight-related concerns, (2) Body } \\
\text { discomfort, and (3) Restraint. }\end{array}$ \\
\hline Zohar et al. [11] & 2017 & Israel & $\begin{array}{c}292 \text { community volunteers } \\
(241 \text { female, } 51 \text { male) } \\
\text { mean age }=33.39(\mathrm{SD}=14.52) \text {. }\end{array}$ & $\begin{array}{l}\text { A three-factor model gave the best } \\
\text { fit with weight and shape concerns } \\
\text { converted into a single factor. }\end{array}$ \\
\hline Machado et al. [35] & 2018 & Portugal & $\begin{array}{l}\text { Two samples: (1) } 2026 \text { high school } \\
\text { students (all female), mean age } 16.2 \\
(\mathrm{SD}=1.34) \text { years, and } 2091 \text { college } \\
\text { students (all female), mean age } 23.8 \\
(\mathrm{SD}=9.16) \text { years, }(2) 609 \text { clinical } \\
\text { participants seeking treatment for an } \\
\text { eating disorder (592 female, } \\
17 \text { male), mean age } \\
23.8(\mathrm{SD}=9.16) \text { years. }\end{array}$ & $\begin{array}{c}\text { A three-factor, seven-item model } \\
\text { suggested by Grilo et al., } 2013 \text { [33] } \\
\text { was found to have the best fit for } \\
\text { both groups. }\end{array}$ \\
\hline Serier et al. [36] & 2018 & USA & $\begin{array}{l}561 \text { university students (all female), } \\
336 \text { Hispanic and } 225 \text { non-Hispanic, } \\
\text { mean age = } 20.11(\mathrm{SD}=3.43)\end{array}$ & $\begin{array}{l}\text { A three-factor seven-item structure } \\
\text { suggested by Grilo et al., } 2013 \text { [33] } \\
\text { was found to have the best fit for } \\
\text { both groups. }\end{array}$ \\
\hline
\end{tabular}


Table 1. Cont.

\begin{tabular}{|c|c|c|c|c|}
\hline Authors & Year & Country & Sample & Factor Solution and Item Number \\
\hline Carey at al. [37] & 2019 & UK & $\begin{array}{c}2459 \text { participants from three } \\
\text { samples: }(1) 1075 \text { students } \\
\text { (851 female, } 224 \text { male), mean age } \\
19.89(\mathrm{SD}=1.98),(2) 653 \text { students } \\
(489 \text { females, } 164 \text { males), mean age } \\
22.16(\mathrm{SD}=3.69) \text { and } 22.86 \\
(\mathrm{SD}=3.69) \text { years respectively, and } \\
(3) 731 \text { non-students }(561 \text { female, } \\
179 \text { male), mean age } 32.68 \\
(\mathrm{SD}=10.25) \text { and } 34.39(\mathrm{SD}=11.08) \\
\text { years respectively. }\end{array}$ & $\begin{array}{l}\text { A three-factor model had the best fit } \\
\text { for both gender using an 18-item for } \\
\text { female and a 16-item model for male } \\
\text { participants: (1) Shape and weight } \\
\text { concern, (2) Preoccupation and } \\
\text { eating concern and (3) Restriction. }\end{array}$ \\
\hline Compte et al. [38] & 2019 & Argentina & $\begin{array}{c}\text { Four samples: (1) } 232 \text { college } \\
\text { students (all male), mean age } 23.53 \\
(\mathrm{SD}=5.61) \text { years, }(2) 286 \\
\text { weightlifters (all male), mean age } \\
29.24(\mathrm{SD}=9.27) \text { years, } 3) 279 \\
\text { cross-fit gym users (all male), mean } \\
\text { age } 29.86(\mathrm{SD}=7.41) \text { years, and } \\
\text { (4) } 203 \text { rugby players (all male), } \\
\text { mean age } 21.71(\mathrm{SD}=3.45) \text { years. }\end{array}$ & $\begin{array}{l}\text { A one-factor, eight-item model was } \\
\text { found to have the best fit between } \\
\text { the groups. }\end{array}$ \\
\hline Sepúlveda et al. [39] & 2019 & Spain & $\begin{array}{l}167 \text { adolescent patients with an } \\
\text { eating disorder (all female), mean } \\
\text { age } 15.45(\mathrm{SD}=1.59) \text { years. }\end{array}$ & $\begin{array}{c}\text { The two-factor structure suggested } \\
\text { by Penelo et al., } 2013 \text { [32] was found } \\
\text { to have the best fit. }\end{array}$ \\
\hline Lewis-Smith et al. [40] & 2020 & India & $\begin{array}{c}1413 \text { adolescents ( } 635 \text { females, } \\
778 \text { males) from an urban private } \\
\text { school, mean age } \\
13(\mathrm{SD}=0.84) \text { years. }\end{array}$ & $\begin{array}{l}\text { A two-factor model, } 15 \text { items for } \\
\text { girls and } 18 \text { for boys, had the best fit } \\
\text { (1) Preoccupation and control, and } \\
\text { (2) Weight and shape concerns }\end{array}$ \\
\hline $\begin{array}{l}\text { Rand-Giovanetti et al. } \\
\text { [41] }\end{array}$ & 2020 & Australia & $\begin{array}{c}940 \text { psychology students } \\
(69.9 \% \text { female, } 29.6 \% \text { male, } 1 \% \\
\text { other), mean age } \\
20.34(\mathrm{SD}=3.74) \text { years. }\end{array}$ & $\begin{array}{l}\text { The four-factor 22-item structure } \\
\text { suggested by Friborg et al. } 2015 \text { was } \\
\text { found to have the best fit: (1) Dietary } \\
\text { restraint, (2) Preoccupation and } \\
\text { restriction, (3) Weight and shape } \\
\text { concern, and (4) Eating shame. }\end{array}$ \\
\hline Scharmer et al. [42] & 2020 & USA & $\begin{array}{l}703 \text { participants from the general } \\
\text { population (all male), mean age } \\
33.76(\mathrm{R}=18-67) \text { years. } 70.2 \% \\
\text { identified as heterosexual while } \\
29.8 \% \text { identified as a sexual minority } \\
\text { (homosexual, bisexual, or queer). }\end{array}$ & $\begin{array}{l}\text { The three-factor seven-item } \\
\text { structure suggested by Grilo et al. } \\
\text { (2013) [33] was found to have the } \\
\text { best fit for both groups. }\end{array}$ \\
\hline Klimek et al. [43] & 2021 & USA & $\begin{array}{c}962 \text { cisgender individuals from a } \\
\text { sexual minority ( } 483 \text { female, } \\
479 \text { male), mean age } \\
23.68(\mathrm{SD}=3.73 \text { ) years. }\end{array}$ & $\begin{array}{l}\text { Two different model were found to } \\
\text { have an adequate fit: A three-factor, } \\
\text { seven-item structure suggested by } \\
\text { Grilo et al. (2013) [33] and a } \\
\text { four-factor, 22-item structure } \\
\text { suggested by Friborg et al., } 2015 \text {. }\end{array}$ \\
\hline Rica et al. [44] & 2021 & Spain & $\begin{array}{l}850 \text { university students (all male), } \\
\text { mean age } 19.8(\mathrm{SD}=2.8 \text { ) years. }\end{array}$ & $\begin{array}{l}\text { A two-factor had the best fit: } \\
\text { (1) Restraint, and (2) Weight and } \\
\text { shape eating concern. }\end{array}$ \\
\hline
\end{tabular}

$\mathrm{SD}$ = standard deviation; $\mathrm{R}$ = range from youngest to oldest age of participants; $\mathrm{BED}=$ Binge Eating Disorder.

Different shorter versions (e.g., seven items and eight items) have been created and suggested, but none have been replicated sufficiently to conclude and recommend new evident factor solutions. 


\subsection{Internal Consistency}

The descriptive characteristics of participants in the empirical studies are presented in Table 2 according to age, gender, Body Mass Index (BMI) and clinical scores.

Table 2. Demographic characteristics, eating-disorder symptoms and depressive symptoms.

\begin{tabular}{|c|c|c|c|c|}
\hline & $\begin{array}{l}\text { Eating Disorder } \\
\text { Patients }(n=101)\end{array}$ & $\begin{array}{c}\text { Binge Eating } \\
\text { Disorder }(n=300)\end{array}$ & $\begin{array}{l}\text { Elite Athletes } \\
\quad(n=404)\end{array}$ & $\begin{array}{c}\text { Amateur Exercisers } \\
\qquad(n=526)\end{array}$ \\
\hline Age range years & $15-64$ & $18-68$ & $15-47$ & $15-70$ \\
\hline Age mean & 27.6 (SD 9.9) & 39.1 (SD 11.5) & 20.0 (SD 4.7) & 29.6 (SD 10.4) \\
\hline Gender female/male & $97 / 4$ & $271 / 29$ & $207 / 197$ & $364 / 162$ \\
\hline Body Mass Index & $\begin{array}{c}22.4 \\
\text { (range } 7.6-54.3)\end{array}$ & $\begin{array}{c}37.8 \\
\text { (range } 16.6-76.2)\end{array}$ & $\begin{array}{c}21.8 \\
\text { (range } 16.6-30.8)\end{array}$ & $\begin{array}{c}23.7 \\
\text { (range } 15.2-42.0)\end{array}$ \\
\hline \multicolumn{5}{|l|}{ EDE-Q } \\
\hline Restraint eating & 3.62 (SD 1.84) & $2.63(\mathrm{SD} 1.30)$ & $0.83(\mathrm{SD} 1.16)$ & $1.28(\mathrm{SD} 1.41)$ \\
\hline Eating concerns & 3.23 (SD 1.56) & 3.56 (SD 1.10) & 0.46 (SD 0.89) & 0.76 (SD 1.12) \\
\hline Shape Concerns & 4.57 (SD 1.40) & 4.60 (SD 0.92) & 1.20 (SD 1.28) & $1.59(\mathrm{SD} 0.16)$ \\
\hline Weight concerns & 4.25 (SD 1.49) & 4.37 (SD 0.91) & 1.08 8SD 1.02) & $1.46(\mathrm{SD} 0.06)$ \\
\hline Global score & 3.92 (SD 1.42) & 3.79 (SD 0.83) & 0.89 (SD 1.02) & 1.25 (SD 0.05) \\
\hline SCOFF $2+$ & $95.9 \%$ & $\mathrm{NO}$ & $17.1 \%$ & $31 \%$ \\
\hline BED-Q total & $\mathrm{NO}$ & 17.17 (SD 3.68) & $\mathrm{NO}$ & $\mathrm{NO}$ \\
\hline Depression mean score & 29.7 (SD 10.6) & 24.8 (SD 9.1) & 8.97 (SD 7.4) & 12.9 (SD 9.7) \\
\hline Mild depression & $13.5 \%$ & $16.3 \%$ & $4.0 \%$ & $6.8 \%$ \\
\hline Moderate depression & $11.5 \%$ & $19.0 \%$ & $2.0 \%$ & $4.4 \%$ \\
\hline Severe depression & $52.1 \%$ & $30.7 \%$ & $2.7 \%$ & $7.8 \%$ \\
\hline
\end{tabular}

$\mathrm{SD}=$ Standard Deviation; NO = Not Available. EDE-Q = Eating Disorder Examination Questionnaire. BED-Q = Binge Eating Disorder Questionnaire.

The Cronbach's alpha coefficient was low, but acceptable, in the eating disorder sample ranging from 0.61 (weight concern scale) to 0.80 (restraint scale). In the combined sport sample, the alpha coefficient ranged from 0.81 (restraint scale) to 0.91 (shape concern scale). See Table 3.

Table 3. Summary of goodness-of-fit statistics.

\begin{tabular}{|c|c|c|c|c|c|c|c|c|c|c|c|c|c|c|}
\hline \multirow[b]{2}{*}{ Samples } & \multicolumn{4}{|c|}{ Cronbach's $\alpha$} & \multicolumn{5}{|c|}{$\begin{array}{l}\text { Confirmatory Factor Analysis with No } \\
\text { Correlated Uniqueness }\end{array}$} & \multicolumn{5}{|c|}{$\begin{array}{l}\text { Confirmatory Factor Analysis with Two } \\
\text { Correlated Uniquenesses between Items } 7 \& 8 \\
\text { and Items } 22 \& 23\end{array}$} \\
\hline & Restraint & $\begin{array}{c}\text { Eating } \\
\text { Concern }\end{array}$ & $\begin{array}{l}\text { Shape } \\
\text { Concern }\end{array}$ & $\begin{array}{l}\text { Weight } \\
\text { Concern }\end{array}$ & $\begin{array}{c}\chi^{2} \\
(\mathrm{df})\end{array}$ & CFI & TLI & $\begin{array}{l}\text { RMSEA } \\
(90 \%-C I)\end{array}$ & SRMR & $x^{2}(\mathrm{df})$ & CFI & TLI & $\begin{array}{l}\text { RMSEA } \\
(90 \%-C I)\end{array}$ & SRMR \\
\hline $\begin{array}{l}\text { Eating } \\
\text { disorder } \\
\text { \& BED }\end{array}$ & 0.779 & 0.677 & 0.768 & 0.608 & $\begin{array}{l}1585 \\
(203)\end{array}$ & 0.660 & 0.614 & $\begin{array}{l}0.130 \\
(0.125 \\
0.136)\end{array}$ & 0.121 & $\begin{array}{l}1104 \\
(201)\end{array}$ & 0.778 & 0.745 & $\begin{array}{l}0.106 \\
(0.100 \\
0.112)\end{array}$ & 0.118 \\
\hline $\begin{array}{c}\text { Elite \& } \\
\text { exercise }\end{array}$ & 0.805 & 0.813 & 0.910 & 0.818 & $\begin{array}{l}2723 \\
(203)\end{array}$ & 0.821 & 0.796 & $\begin{array}{l}0.116 \\
(0.112 \\
0.119)\end{array}$ & 0.067 & $\begin{array}{l}1777 \\
(201)\end{array}$ & 0.888 & 0.871 & $\begin{array}{l}0.092 \\
(0.088 \\
0.096)\end{array}$ & 0.064 \\
\hline
\end{tabular}

BED = Binge Eating Disorder. CFI = Comparative Fit Index. TLI = Tucker-Lewis Index. RMSEA = Root-Mean-Square Error of Approximation. SRMR = Standardized Root-Mean-square Residual.

The results of the CFA showed no optimal fit of the original four-factor model in the two pooled samples, as the only acceptable test was SRMR in the athlete sample. Accepting correlations between items 7 and 8 and items 22 and 23 that are specifically closely related did not change the results significantly, despite showing a better fit.

Sensitivity analyses with item 8 attributed to the shape concern factor resulted in very similar results. 


\subsection{Construct Validity}

When examining the construct validity of EDE-Q by comparing the relationship between EDE-Q global scale and SCOFF total score (or BED-Q for the BED sample), the results demonstrated positive and significant correlations in all samples. Further, we examined the relationship between EDE-Q and MDI, and found positive and significant correlations in all samples. The results and level of significance are shown in Table 4.

Table 4. Pearson and Spearman correlations between EDE-Q global score and other measures of eating pathology or depression.

\begin{tabular}{|c|c|c|c|c|}
\hline \multirow[b]{2}{*}{ Measure } & \multicolumn{4}{|c|}{ Correlation with EDE-Q Global Score in the Four Samples } \\
\hline & $\begin{array}{l}\text { Mixed Eating } \\
\text { Disorders }\end{array}$ & $\begin{array}{c}\text { Binge Eating } \\
\text { Disorder }\end{array}$ & Elite Athletes & $\begin{array}{l}\text { Regular } \\
\text { Exercisers }\end{array}$ \\
\hline SCOFF & $\begin{array}{c}\mathrm{R}=0.599 \\
p<0.001 \\
\mathrm{Rho}=0.536 \\
p<0.001\end{array}$ & NA & $\begin{array}{c}\mathrm{R}=0.729 \\
p<0.001 \\
\mathrm{Rho}=0.611 \\
p<0.001\end{array}$ & $\begin{array}{c}\mathrm{R}=0.765 \\
p<0.001 \\
\text { Rho }=0.730 \\
p<0.001\end{array}$ \\
\hline BED-Q & NA & $\begin{array}{c}\mathrm{R}=0.347 \\
p<0.001 \\
\text { Rho }=0.335 \\
p<0.001\end{array}$ & NA & NA \\
\hline MDI & $\begin{array}{c}\mathrm{R}=0.606 \\
p<0.001 \\
\mathrm{Rho}=0.574 \\
p<0.001\end{array}$ & $\begin{array}{c}\mathrm{R}=0.451 \\
p<0.001 \\
\mathrm{Rho}=0.454 \\
p<0.001\end{array}$ & $\begin{array}{c}\mathrm{R}=0.569 \\
p<0.001 \\
\mathrm{Rho}=0.499 \\
p<0.001\end{array}$ & $\begin{array}{c}\mathrm{R}=0.686 \\
p<0.001 \\
\mathrm{Rho}=0.595 \\
p<0.001\end{array}$ \\
\hline
\end{tabular}

\section{Discussion}

\subsection{Literature Review and Factor Structure of EDE-Q}

In accordance with other validation studies our Danish study could not empirically support the original factor structure (restraint eating, eating concern, shape concern and weight concern) presented as a theoretical model by Fairburn [1].

The results from the EDE-Q literature across several countries (Table 1) indicate that no evident and consistent factor structure of the original EDE-Q can be identified. Models suggesting one-factor, two-factor and three-factor structures have been presented and evaluated. Shorter versions of EDE-Q have been developed, but the internal structure of the scale differs across studies and samples. Several studies point at a seven-item scale, but also 8 items, 15 items (for female), 16 items (for male), 18 items and 22 items have been proposed during the last 10 years.

The scale was not originally developed from empirical research, but is a theoretical construct that is reasonable from a clinical perspective. The confusion of results may be related to the interpretation of constructs by different populations. Restraint eating can be part of a healthy diet in general populations or in athletic settings, while it is pathological in eating-disorder patients. Weight concerns may be relevant in severely overweight BED patients, while it they are related to disturbed body image in AN patients. Eating concerns may appear differently in clinical eating disorder samples compared to athletic samples. Darcy et al. also suggested that athletes may differ in diet and eating concerns compared to sedentary people [21].

Furthermore, a large number of the elite athletes in the current study reported a 0 -score on several items, leading to a high Cronbach's Alpha coefficient but a weak factor model. The wording of weight and shape concerns may overlap and can be difficult to separate, leading to similar scores that confuse the CFA model, because some items (e.g., 7 and 8 ) are loading on two factors.

Despite the lack of psychometric evidence, the four constructs might be useful in a clinical setting if patients find them coherent. The face validity of EDE-Q and EDE-I may be 
high in the assessment of pathological thoughts and behaviors in collaborations between clinicians and patients. For athletes, the EDE-Q may increase the awareness of disturbed eating patterns and body image. Qualitative research investigating the interpretation of items and factors is recommended in future research.

Further studies of the factor structure may confuse the landscape of psychometric reliability. Instead, we should evaluate the validity of EDE-Q because the instrument may be useful in identifying eating disorders.

\subsection{Validity of the EDE-Q}

Despite lack of internal consistency, the construct validity was confirmed in all samples, as EDE-Q scores were highly correlated with other eating-disorder measures and depressive symptoms. This is in accordance with the American and Hebrew validations of the EDE-Q demonstrating positive and significant correlations between EDE-Q and other health measures, including depressive symptoms [33] and eating disorder symptoms [11]. The German 8-item scale also showed good construct validity with significant positive correlations between EDE-Q and the Eating Attitudes Test [17].

In conclusion, the studies support the ability of the EDE-Q to cover the construct of eating disorders, making it a valid tool for identifying eating disorders, though the lack of evidence of the four-factor model should be considered.

\subsection{Limitations}

It may be a limitation that the four sub-samples in our study differed according to sample size, age, gender, BMI and recruitment method.

Limitations must be taken as the study comprise heterogeneous samples of patients and amateur exercisers which might have affected the results. On the other hand, the factor structure ideally ought to be the same if the EDE-Q should be suggested as a solid questionnaire.

We have chosen a wide range of ED's from AN with very low BMI to BED with very high BMI. Although, this per see increases the external validity of the work, few extreme patients potentially may distort the statistics.

The EDE-Q has been questioned in male samples, and there is a risk of measurement bias as the scale may not comprehensively assess all domains of disordered eating relevant to males [45].

The study might have benefited from adding a fifth group of community controls. However, the EDE-Q was developed to assess symptoms in a clinical setting and risk groups and testing the factor structure in these groups was considered first step.

Despite these concerns, the psychometric properties of the scale were similar across sub-samples, indicating that it is useful across diagnoses and in sport settings.

An exploratory analysis or another CFA model could have accounted for the inability of our CFA to provide an optimal fit of the original four-factor structure. However, the aim of our study was only to validate the existing gold standard scale in Danish and not to explore if another structure could be supported.

It should be mentioned as a potential bias that some of our data were collected during the COVID-19 pandemic. A retrospective study from Italy [46] found that ED symptoms increased in ED patients during March and April 2020. However, the crisis was worse in Italy than in Denmark, including more restrictions and more impact on hospitals, public health and mortality.

\section{Conclusions}

The psychometric evaluation of the EDE-Q in a Danish sample of eating-disorder patients and a sample of athletes did not find evidence for the original four-factor structure. Our literature review further depicted the variance regarding number of factors (one, two or three) and items suggested (from 7 to 22) in previous validation studies. Thus, the 
four-factor internal structure of EDE-Q may be understood as a theoretical model useful in clinical settings, but it is not supported by empirical data.

However, the construct validity was high in all samples, indicating that the scale has the potential to identify individuals with eating pathology and comorbid mental distress. Development of a sport-specific tool may present with higher reliability and validity. Until then, the EDE-Q is a usable screening tool, but with caution and awareness of its limitations.

Author Contributions: M.B.L., conceptualization, methodology, validation, formal analysis, investigation, resources, data curation, writing - original draft preparation, writing-review and editing. L.H., project administration, data curation, writing — original draft preparation, writing-review and editing. K.K.J., project administration, formal analysis, writing-review and editing. P.V.L., software, formal analysis, data curation, writing-original draft preparation. L.C., supervision, methodology, formal analysis, writing - review and editing. J.B.B., project administration, methodology, writingoriginal draft preparation, data curation. R.K.S., formal analysis, supervision, writing-review and editing. J.L., conceptualization, methodology, validation, writing-original draft preparation. All authors have read and agreed to the published version of the manuscript.

Funding: This research has not received any funding.

Institutional Review Board Statement: The study was conducted according to the guidelines of the Declaration of Helsinki, and the Ethics Committee of the Region of Southern Denmark (case number 2021200-57 and 20192000-145) confirmed that Danish survey studies do not need ethical approval.

Informed Consent Statement: Informed consent was obtained from all subjects involved in the study.

Data Availability Statement: The data presented in this study are available by request.

Acknowledgments: In this section, you can acknowledge any support given which is not covered by the author contribution or funding sections. This may include administrative and technical support, or donations in kind (e.g., materials used for experiments).

Conflicts of Interest: The authors declare no conflict of interest.

\section{Appendix A. Binge Eating Disorder Questionnaire (BED-Q)}

Please indicate how many binge eating episodes per week you have had during the past three months.

In a binge eating episode you eat a larger amount of food in a short period of time (e.g., within two hours) than most people would, and you lose control over your eating.

Check (" $X$ ") the number of binge eating episodes you have had per week.

Indicate the average number of binge eating episodes, if it varies how many binge eating episodes you have had during the past three months (e.g., 20 episodes in 12 weeks $=1-3$ episodes per week).

If you have not had any binge eating episodes in the past three months, please check "No" in all questions.

Remember to answer all questions (1-9).

\begin{tabular}{|c|c|c|c|c|c|c|}
\hline $\begin{array}{c}\text { In the Past Three Months, } \\
\text { Have You: }\end{array}$ & No & $\begin{array}{c}\text { Yes, Less } \\
\text { Than Once a } \\
\text { Week }\end{array}$ & $\begin{array}{c}\text { Yes, } \\
\text { 1-3 Times per } \\
\text { Week }\end{array}$ & $\begin{array}{c}\text { Yes, } \\
\text { 4-7 Times } \\
\text { per Week }\end{array}$ & $\begin{array}{c}\text { Yes, } \\
\text { 8-13 Times } \\
\text { per Week }\end{array}$ & $\begin{array}{c}\text { Yes, } \\
14 \text { or More } \\
\text { Times per Week }\end{array}$ \\
\hline $\begin{array}{l}\text { 1. Had binge eating episodes? } \\
\text { (i.e., in a short period of time } \\
\text { (e.g., two hours) eaten a larger } \\
\text { amount of food than what most } \\
\text { people would)? }\end{array}$ & & & & & & \\
\hline $\begin{array}{l}\text { 2. Lost control over your eating } \\
\text { during these episodes? (e.g., } \\
\text { that you could not stop eating or } \\
\text { control how much you were } \\
\text { eating)? }\end{array}$ & & & & & & \\
\hline
\end{tabular}




\begin{tabular}{|c|c|c|c|c|c|c|}
\hline $\begin{array}{c}\text { In the Past Three Months, } \\
\text { Have You: }\end{array}$ & No & $\begin{array}{c}\text { Yes, Less } \\
\text { Than Once a } \\
\text { Week }\end{array}$ & $\begin{array}{l}\text { Yes, } \\
\text { 1-3 Times per } \\
\text { Week }\end{array}$ & $\begin{array}{l}\text { Yes, } \\
\text { 4-7 Times } \\
\text { per Week }\end{array}$ & $\begin{array}{c}\text { Yes, } \\
\text { 8-13 Times } \\
\text { per Week }\end{array}$ & $\begin{array}{c}\text { Yes, } \\
14 \text { or More } \\
\text { Times per Week }\end{array}$ \\
\hline \multicolumn{7}{|l|}{$\begin{array}{l}\text { 3. Had binge eating episodes, } \\
\text { where you were eating much } \\
\text { faster rapidly than normal? }\end{array}$} \\
\hline \multicolumn{7}{|l|}{$\begin{array}{l}\text { 4. Had binge eating episodes, } \\
\text { where you were eating until you } \\
\text { were uncomfortably full? }\end{array}$} \\
\hline \multicolumn{7}{|l|}{$\begin{array}{l}\text { 5. Had binge eating episodes, } \\
\text { where you were eating large } \\
\text { amounts of food, even though } \\
\text { you were not hungry? }\end{array}$} \\
\hline \multicolumn{7}{|l|}{$\begin{array}{l}\text { 6. Had binge eating episodes, } \\
\text { where you were eating alone, } \\
\text { because you were embarrassed } \\
\text { about, how much you ate? }\end{array}$} \\
\hline \multicolumn{7}{|l|}{$\begin{array}{c}\text { 7. Had binge eating episodes, } \\
\text { where you felt disgusted with } \\
\text { yourself, depressed or guilty } \\
\text { afterwards? }\end{array}$} \\
\hline \multicolumn{7}{|l|}{$\begin{array}{l}\text { 8. Vomited after episodes of } \\
\text { binge eating? }\end{array}$} \\
\hline $\begin{array}{l}\text { 9. Are you very distressed by } \\
\text { the binge eating episodes? }\end{array}$ & Yes & & & No & & \\
\hline
\end{tabular}

\section{BED diagnostic score (see gray cells in the table below)}

- Both question 1 and 2 are 2 or higher.

- At least three of the questions from 3 to 7 are 2 or higher.

- Question 8 is "No" or "Yes, less than once a week".

- Question 9 is "Yes".

\begin{tabular}{|c|c|c|c|c|c|c|}
\hline $\begin{array}{c}\text { In the Past Three Months, } \\
\text { Have You: }\end{array}$ & No & $\begin{array}{c}\text { Yes, Less } \\
\text { Than once a } \\
\text { Week }\end{array}$ & $\begin{array}{l}\text { Yes, } \\
\text { 1-3 Times per } \\
\text { Week }\end{array}$ & $\begin{array}{l}\text { Yes, } \\
\text { 4-7 Times } \\
\text { per Week }\end{array}$ & $\begin{array}{l}\text { Yes, } \\
\text { 8-13 Times } \\
\text { per Week }\end{array}$ & $\begin{array}{c}\text { Yes, } \\
\text { 14 or More } \\
\text { Times per Week }\end{array}$ \\
\hline $\begin{array}{l}\text { 1. Had binge eating episodes? } \\
\text { (i.e., in a short period of time } \\
\text { (e.g., two hours) eaten a larger } \\
\text { amount of food than what most } \\
\text { people would)? }\end{array}$ & 0 & 1 & 2 & 3 & 4 & 5 \\
\hline $\begin{array}{l}\text { 2. Lost control over your eating } \\
\text { during these episodes? (e.g., } \\
\text { that you could not stop eating or } \\
\text { control how much you were } \\
\text { eating)? }\end{array}$ & 0 & 1 & 2 & 3 & 4 & 5 \\
\hline $\begin{array}{l}\text { 3. Had binge eating episodes, } \\
\text { where you were eating much } \\
\text { faster rapidly than normal? }\end{array}$ & 0 & 1 & 2 & 3 & 4 & 5 \\
\hline $\begin{array}{l}\text { 4. Had binge eating episodes, } \\
\text { where you were eating until you } \\
\text { were uncomfortably full? }\end{array}$ & 0 & 1 & 2 & 3 & 4 & 5 \\
\hline
\end{tabular}




\begin{tabular}{|c|c|c|c|c|c|c|}
\hline $\begin{array}{c}\text { In the Past Three Months, } \\
\text { Have You: }\end{array}$ & No & $\begin{array}{c}\text { Yes, Less } \\
\text { Than once a } \\
\text { Week }\end{array}$ & $\begin{array}{l}\text { Yes, } \\
\text { 1-3 Times per } \\
\text { Week }\end{array}$ & $\begin{array}{l}\text { Yes, } \\
\text { 4-7 Times } \\
\text { per Week }\end{array}$ & $\begin{array}{l}\text { Yes, } \\
\text { 8-13 Times } \\
\text { per Week }\end{array}$ & $\begin{array}{c}\text { Yes, } \\
14 \text { or More } \\
\text { Times per Week }\end{array}$ \\
\hline $\begin{array}{l}\text { 5. Had binge eating episodes, } \\
\text { where you were eating large } \\
\text { amounts of food, even though } \\
\text { you were not hungry? }\end{array}$ & 0 & 1 & 2 & 3 & 4 & 5 \\
\hline $\begin{array}{l}\text { 6. Had binge eating episodes, } \\
\text { where you were eating alone, } \\
\text { because you were embarrassed } \\
\text { about, how much you ate? }\end{array}$ & 0 & 1 & 2 & 3 & 4 & 5 \\
\hline $\begin{array}{c}\text { 7. Had binge eating episodes, } \\
\text { where you felt disgusted with } \\
\text { yourself, depressed or guilty } \\
\text { afterwards? }\end{array}$ & 0 & 1 & 2 & 3 & 4 & 5 \\
\hline $\begin{array}{l}\text { 8. Vomited after episodes of } \\
\text { binge eating? }\end{array}$ & 0 & 1 & 2 & 3 & 4 & 5 \\
\hline $\begin{array}{l}\text { 9. Are you very distressed by } \\
\text { the binge eating episodes? }\end{array}$ & Yes & & & Not & & \\
\hline
\end{tabular}

\section{BED severity score}

Add question 1-7 (0-5). Question 8 is not included:

$0=$ No BED symptoms

1-9 = Sub-clinical BED symptoms

$10-14=$ Possible indication of mild BED

$15-21=$ Possible indication of moderate BED

22-28 = Possible indication of severe BED

29-35 = Possible indication of extreme BED

Minimum is 0; maximum is 35

\section{References}

1. Fairburn, C.G.; Beglin, S.J. Assessment of eating disorders: Interview or self-report questionnaire? Int. J. Eat. Disord. 1994, 16, 363-370.

2. Berg, K.C.; Peterson, C.B.; Frazier, P.; Crow, S.J. Psychometric evaluation of the eating disorder examination and eating disorder examination-questionnaire: A systematic review of the literature: Psychometrics of the EDE and EDE-Q. Int. J. Eat. Disord. 2012, 45, 428-438. [CrossRef]

3. Aardoom, J.J.; Dingemans, A.E.; Op't Landt, M.C.S.; Van Furth, E.F. Norms and discriminative validity of the Eating Disorder Examination Questionnaire (EDE-Q). Eat. Behav. 2012, 13, 305-309. [CrossRef]

4. Peláez-Fernández, M.A.; Javier Labrador, F.; Raich, R.M. Validation of eating disorder examination questionnaire (EDE-Q)Spanish version-For screening eating disorders. Span. J. Psychol. 2012, 15, 817-824. [CrossRef]

5. Peláez-Fernández, M.A.; Labrador, J.F.; Raich, R.M. Norms for the Spanish version of the Eating Disorders Examination Questionnaire (S-EDE-Q). Psicothema 2013, 25, 107-114.

6. Villarroel, A.M.; Penelo, E.; Portell, M.; Raich, R.M. Screening for Eating Disorders in Undergraduate Women: Norms and Validity of the Spanish Version of the Eating Disorder Examination Questionnaire (EDE-Q). J. Psychopathol. Behav. Assess. 2011, 33, 121-128. [CrossRef]

7. Mitsui, T.; Yoshida, T.; Komaki, G. Psychometric properties of the eating disorder examination-questionnaire in Japanese adolescents. Biopsychosoc. Med. 2017, 11, 9. [CrossRef]

8. Yucel, B.; Polat, A.; Ikiz, T.; Dusgor, B.P.; Elif Yavuz, A.; Sertel Berk, O. The Turkish Version of the Eating Disorder Examination Questionnaire: Reliability and Validity in Adolescents: The Turkish Version of the EDE-Q. Eur. Eat. Disord. Rev. 2011, 19, 509-511. [CrossRef]

9. Mahmoodi, M.; Moloodi, R.; Ghaderi, A.; Babai, Z.; Saleh, Z.; Alasti, H.; Naghashian, F.; Mohammadpour, Z. The Persian Version of Eating Disorder Examination Questionnaire and Clinical Impairment Assessment: Norms and Psychometric Properties for Undergraduate Women. Iran. J. Psychiatry 2016, 11, 67-74. [PubMed]

10. Isomaa, R.; Lukkarila, I.-L.; Ollila, T.; Nenonen, H.; Charpentier, P.; Sinikallio, S.; Karhunen, L. Development and preliminary validation of a Finnish version of the Eating Disorder Examination Questionnaire (EDE-Q). Nord. J. Psychiatry 2016, 70, 542-546. [CrossRef] [PubMed] 
11. Zohar, A.H.; Lev-Ari, L.; Bachner-Melman, R. The EDE-Q in Hebrew: Structural and Convergent/Divergent Validity in a Population Sample. Isr. J. Psychiatry Relat. Sci. 2017, 54, 15-20. [PubMed]

12. Giovazolias, T.; Tsaousis, I.; Vallianatou, C. The factor structure and psychometric properties of the Greek version of the Eating Disorders Examination Questionnaire (EDE-Q). Eur. J. Psychol. Assess. 2013, 29, 189-196. [CrossRef]

13. Reas, D.L.; Wisting, L.; Kapstad, H.; Lask, B. Convergent Validity of the Eating Disorder Examination and the Eating Disorder Examination-Questionnaire Among University Women in Norway. Eur. Eat. Disord Rev. 2011, 19, 357-361. [CrossRef] [PubMed]

14. Rø, Ø.; Reas, D.L.; Stedal, K. Eating Disorder Examination Questionnaire (EDE-Q) in Norwegian adults: Discrimination between female controls and eating disorder patients. Eur. Eat. Disord. Rev. 2015, 23, 408-412. [CrossRef]

15. Forsén Mantilla, E.; Birgegård, A.; Clinbton, D. Factor analyses of the adolescent version of the Eating Disorder Examination Questionnaire (EDE-Q): Results from Swedish general population and clinical samples. J. Eat. Disord. 2017, 5, 19. [CrossRef] [PubMed]

16. Carrard, I.; Rebetez, M.M.L.; Mobbs, O.; Van der Linden, M. Factor Structure of a French version of the eating disorder examination-questionnaire among women with and without binge eating disorder symptoms. Eat. Weight Disord. 2015, 20, 137-144. [CrossRef] [PubMed]

17. Kliem, S.; Mössle, T.; Zenger, M.; Strauss, B.; Brähler, E.; Hilbert, A. The Eating Disorder Examination-Questionnaire 8: A Brief Measure of Eating Disorder Psychopathology (EDE-Q8). Int. J. Eat. Disord. 2016, 49, 613-616. [CrossRef]

18. Kjeldbjerg, M.L.; Clausen, L.; Højgaard, D.R.M.A. Factor analysis of the eating disorder examination in a sample of female adolescents and adults with eating disorders. Int. J. Eat. Disord. 2021, 54, 879-886. [CrossRef] [PubMed]

19. Sundgot-Borgen, J.; Torstveit, M.K. Prevalence of eating disorders in elite athletes is higher than in the general population. Clin. J. Sport Med. 2004, 14, 25-32. [CrossRef]

20. Martinsen, M.; Bratland-Sanda, S.; Eriksson, A.K.; Sundgot-Borgen, J. Dieting to win or to be thin? A study of dieting and disordered eating among adolescent elite athletes and non-athlete controls. Br. J. Sports Med. 2010, 44, 70-76. [CrossRef]

21. Darcy, A.M.; Hardy, K.K.; Crosby, R.D.; Lock, J.; Peebles, R. Factor structure of the Eating Disorder Examination Questionnaire (EDE-Q) in male and female college athletes. Body Image 2013, 10, 399-405. [CrossRef]

22. Lichtenstein, M.B.; Melin, A.; Szabo, A.; Holm, L. The prevalence of exercise addiction symptoms in elite athletes. Front. Sports Act. Living 2021, 3, 160. [CrossRef] [PubMed]

23. Elsass, P.; Ivanouw, J.; Mortensen, E.L.; Poulsen, S.; Rosenbaum, B. Assessmentmetoder—Håndbog for Psykologer og Psykiatere, 1st ed.; Dansk Psykologisk Forlag: Copenhagen, Denmark, 2006; pp. 55-78.

24. Morgan, J.F.; Reid, F.; Lacey, J.H. The SCOFF questionnaire. West. J. Med. 2001, 172, 164-165. [CrossRef] [PubMed]

25. Botella, J.; Sepúlveda, A.R.; Huang, H.; Gambara, H. A meta-analysis of the diagnostic accuracy of the SCOFF. Span. J. Psychol. 2012, 16, 92. [CrossRef]

26. Lichtenstein, M.B.; Hemmingsen, S.D.; Støving, R.K. Identification of eating disorder symptoms in Danish adolescents with the SCOFF questionnaire. Nord. J. Psychiatry 2017, 71, 340-347. [CrossRef]

27. American Psychiatric Association. Chapter 13. Feeding and Eating Disorders. In Diagnostic and Statistical Manual of Mental Disorders, 5th ed.; American Psychiatric Association: Arlington, VA, USA, 2013.

28. Bech, P.; Rasmussen, N.A.; Olsen, L.R.; Noerholm, V.; Abildgaard, W. The sensitivity and specificity of the Major Depression Inventory, using the Present State Examination as the index of diagnostic validity. J. Affect. Disord. 2001, 66, 159-164. [CrossRef]

29. Bech, P.; Timmerby, N.; Martiny, K.; Lunde, M.; Soendergaard, S. Psychometric evaluation of the Major Depression Inventory (MDI) as depression severity scale using the LEAD (Longitudinal Expert Assessment of All Data) as index of validity. BMC Psychiatry 2015, 5, 190. [CrossRef]

30. Cuijpers, P.; Dekker, J.; Noteboom, A.; Smits, N.; Peen, J. Sensitivity and specificity of the Major Depression Inventory in outpatients. BMC Psychol. 2007, 7, 39. [CrossRef]

31. Barnes, J.; Prescott, T.; Muncer, S. Confirmatory factor analysis for the Eating Disorder Examination Questionnaire: Evidence supporting a three-factor structure. Eat. Behav. 2012, 13, 379-381. [CrossRef]

32. Penelo, E.; Negrete, A.; Portell, M.; Raich, R.M. Psychometric properties of the Eating Disorder Examination Questionnaire (EDE-Q) and norms for rural and urban adolescent males and females in Mexico. PLoS ONE 2013, 8, e83245. [CrossRef]

33. Grilo, C.M.; Reas, C.M.; Hopwood, C.J.; Crosby, R.D. Factor Structure and Construct Validity of the Eating Disorder ExaminationQuestionnaire in College Students: Further Support for a Modified Brief Version. Int. J. Eat. Disord. 2014, 48, 284-289. [CrossRef]

34. White, H.J.; Haycraft, E.; Goodwin, H.; Meyer, C. Eating disorder examination questionnaire: Factor structure for adolescent girls and boys. Int. J. Eat. Disord. 2014, 47, 99-104. [CrossRef]

35. Machado, P.P.P.; Grilo, C.M.; Crosby, R.D. Replication of a Modified Factor Structure for the Eating Disorder ExaminationQuestionnaire: Extension to Clinical Eating Disorder and Non-clinical Samples in Portugal. Eur. Eat. Disord. Rev. 2018, 26, 75-80. [CrossRef]

36. Serier, K.N.; Smith, J.E.; Yeater, E.A. Confirmatory factor analysis and measurement invariance of the Eating Disorder Examination Questionnaire (EDE-Q) in a non-clinical sample of non-Hispanic White and Hispanic women. Eat. Behav. 2018, 31, 53-59. [CrossRef]

37. Carey, M.; Kupeli, N.; Knight, R.; Troop, N.A.; Jenkinson, P.M.; Preston, C. Eating Disorder Examination Questionnaire (EDE-Q): Norms and psychometric properties in U.K. females and males. Psychol. Assess 2019, 31, 839-850. [CrossRef] 
38. Compte, E.J.; Nagata, J.M.; Sepúlveda, A.R.; Schweiger, S.; Sbdar, L.S.; Silva, B.C.; Bressan, M.; Rivas, A.; Menga, S.; Cortes, C.; et al. Confirmatory factor analysis and measurement invariance of the eating disorders examination-questionnaire across four male samples in Argentina. Int. J. Eat. Disord. 2019, 52, 740-745. [CrossRef]

39. Sepúlveda, A.R.; Compte, E.J.; Faya, M.; Villaseñor, A.; Gutierrez, S.; Andrés, P.; Graell, M. Spanish validation of the Eating Disorder Examination Questionnaire for Adolescents (EDE-Q-A): Confirmatory factor analyses among a clinical sample. Eat. Disord. 2019, 27, 565-576. [CrossRef]

40. Lewis-Smith, H.; Garbett, K.M.; Chaudhry, A.; Uglik-Marucha, N.; Vitoratou, S.; Dhillon, M.; Shroff, H.; Diedrichs, P.C. Adaptation and validation of the Eating Disorder Examination-Questionnaire in English among urban Indian adolescents. Int. J. Eat. Disord. 2021, 54, 87-202. [CrossRef]

41. Rand-Giovannetti, D.; Cicero, D.C.; Mond, J.M.; Latner, J.D. Psychometric Properties of the Eating Disorder ExaminationQuestionnaire (EDE-Q): A Confirmatory Factor Analysis and Assessment of Measurement Invariance by Sex. Assessment 2020, 27, 164-177. [CrossRef]

42. Scharmer, C.; Donahue, J.M.; Heiss, S.; Anderson, D.A. Factor structure of the Eating Disorder Examination-Questionnaire among heterosexual and sexual minority males. Eat. Behav. 2020, 38, 101403. [CrossRef]

43. Klimek, P.; Convertino, A.D.; Pennesi, J.L.; Gonzales, M., 4th; Roesch, S.C.; Nagata, J.M.; Blashill, A.J. Confirmatory factor and measurement invariance analyses of the Eating Disorder Examination Questionnaire in sexual minority men and women. Int. J. Eat. Disord. 2021, 54, 745-754. [CrossRef]

44. Rica, R.; Solar, M.; Compte, E.J.; Sepúlveda, A.R. Establishing the optimal male cut-off point: Confirmatory factor analysis of the eating disorder examination-questionnaire (EDE-Q) in a representative sample of Spanish university students. Eat. Weight Disord. 2021, 2, 88 .

45. Schaefer, L.M.; Smith, K.E.; Leonard, R.; Wetterneck, C.; Smith, B.; Farrell, N.; Riemann, B.C.; Frederick, D.A.; Schaumberg, K.; Klump, K.L.; et al. Identifying a male clinical cutoff on the Eating Disorder Examination-Questionnaire (EDE-Q). Int. J. Eat. Disord. 2018, 51, 1357-1360. [CrossRef]

46. Monteleone, A.M.; Cascino, G.; Marciello, F.; Abbate-Daga, G.; Baiano, M.; Balestrieri, M.; Barone, E.; Bertelli, S.; Carpiniello, B.; Castellini, G.; et al. Risk and resilience factors for specific and general psychopathology worsening in people with Eating Disorders during COVID-19 pandemic: A retrospective Italian multicentre study. Eat Weight Disord. Stud. Anorex. Bulim. Obesity 2021, 8, 1791. [CrossRef] 